\title{
PTU-026 THE PREVALENCE AND SIGNIFICANCE OF ANAEMIA IN PATIENTS RECEIVING NEO-ADJUVANT CHEMO-RADIOTHERAPY FOR TREATMENT OF RECTAL CARCINOMA
}

doi:10.1136/gut.2011.239301.154

L Bell, ${ }^{*}$ C J Walter, C Jackson, N Borley, A Goodman, J M Wheeler ${ }^{1}$ Colorectal Surgery, Cheltenham General Hospital, Cheltenham, UK

Introduction Previous studies have shown that anaemia during neo-adjuvant chemo-radiotherapy treatment (NCRT) for rectal carcinoma is associated with reduced down-staging when assessed histologically using the Rectal Cancer Regression Group (RCRG). ${ }^{1}$

The aim of this study was to assess the prevalence and significance of anaemia during NCRT for rectal cancer at our centre.

Methods Hospital coding databases were used to identify all patients undergoing NCRT for rectal cancer at our centre between start of 2004 and end of 2009. A retrospective review of computerised haematology and histology records was used to extract and record: patient details; haemoglobin $(\mathrm{Hb})$ levels throughout NCRT and RCRGs (a 3-point scale where grade 1 represents the greatest histological regression and grade 3 the least). Anaemia was defined by our laboratory's standards ( $\mathrm{Hb}<11.5 \mathrm{~g} / \mathrm{dl}$ and $<13 \mathrm{~g} / \mathrm{dl}$ for women and men respectively). Results were analysed using GnuPSPP statistical software.

Results 24 (34\%) of the 70 patients (51 male, 19 female) with a median age of 66 (IOR 60-72.75) treated with NCRT during the study period were shown to be anaemic. Two patients (3\%) had no Hb's recorded.

Anaemic patients demonstrated significantly higher RCRG than non-anaemic patients with $17 \%, 38 \%, 46 \%$ versus $59 \%$, $30 \%, 11 \%$ for RCRG $1-3$ respectively $p<0.001$.

Histology results from patients with lower RCRGs demonstrated lower T scores $(\mathrm{p}<0.01)$ from the TNM staging and a trend to lower $\mathrm{N}$ scores $(\mathrm{p}=0.06)$.

Conclusion At our centre the prevalence of anaemia in patients undergoing NCRT is $34 \%$. Anaemia during NCRT in this study is associated with significant reductions in downstaging as demonstrated by higher RCRGs, which in turn, is associated with worse TNM staging.

Competing interests None.

Keywords anaemia, radiotherapy, rectal cancer.

\section{REFERENCE}

1. Box B, Lindsey I, Wheeler J, et al. Neoadjuvant therapy for rectal cancer: improved tumour suppression, local recurrence and overall survival in the nonanaemic patient. Dis Colon Rectum 2005;48:1153-60. 